\title{
A COMPARISON OF THE FEEDING MECHANISMS OF CALMA GLAUCOIDES AND NEBALIOPSIS TYPICA
}

\author{
By H. G. Q. Rowett, \\ Technical College, Plymouth
}

(Text-figs. I-3)

INTRODUCTION

The feeding mechanism of the nudibranch Calma glaucoides (Alder \& Hancock) has been described by Evans (1922) and that of the crustacean Nebaliopsis typica (Sars) by Cannon (193I) and myself(1943). Calma is seasonally relatively abundant in the coastal waters of parts of Europe where it is found attached to the eggs of certain fishes. It is known to feed exclusively on these eggs and young embryos. Nebaliopsis is a deep pelagic form from the Antarctic Ocean. It has been obtained only rarely and cannot be observed alive and in its natural environment. Two specimens were available for examination. Thus complete certainty about the diet is impossible. The external features and mouth parts were described by Cannon and the structure of these suggested that Nebaliopsis, like other known Nebaliacea, might be a filter feeder. Examination of the morphology of the gut and its contents indicates that this is very improbable. There is no mechanism for the sorting out of particles of different sizes such as is found in other filter feeders. The food passes into a blind sac and there is no apparent means by which the large quantities of indigestible material taken in when particles are filtered from the water indiscriminately might be evacuated. In addition to this negative evidence the food mass found in the large storage chamber of one specimen was completely homogeneous and structureless, its appearance in section suggesting coagulated yolk. It has therefore been suggested that Nebaliopsis feeds on fish eggs found floating in the deeper water layers. Some eggs were caught along with all the specimens of Nebaliopsis which have so far been obtained.

Both Calma and Nebaliopsis have evolved certain peculiar characteristics in which they differ from their nearest relatives and which may be functionally associated with this specialized type of food. Thus these two species, one a mollusc and the other a crustacean, provide an interesting example of parallel adaptation.

As both species have already been described in detail the descriptions and diagrams given here are intended only to clarify the comparison which follows.

I wish to thank Prof. H. Graham Cannon, F.R.S., for drawing my attention to Calma and for his criticism and advice. 


\section{CALMA GLAUCOIDES}

Cálma feeds on eggs of various species of shore living fish, which are laid on the sea floor in coastal waters. Once the eggs have been found Calma crawls on to them and, according to Evans, sticks tightly by means of its 'face' which 'fits like a hood over the egg'. It sucks a part of the egg membranes into its mouth by the action of the powerful retractor muscles of the buccal mass (Fig. 2, A, B, ret). These muscles enlarge the buccal cavity and at the same time draw the jaws out laterally while the retractor muscles of the odontophore draw the radula diagonally upwards and backwards. The ridges on the lips and oral hood grip the slippery surface of the egg while the jaws are brought together by bands of muscle which stretch between them on their ventral sides, connecting them together along the whole of their length except in the region of the mouth (Fig. $2 \mathrm{~B}$, vent). Muscles interwoven with the retractors serve to keep the biting edges of the jaws tilted towards the mouth (Fig. 2, A, B, j.m). Simultaneously the radula is lowered to meet the jaws and may be protruded out of the mouth. This movement is

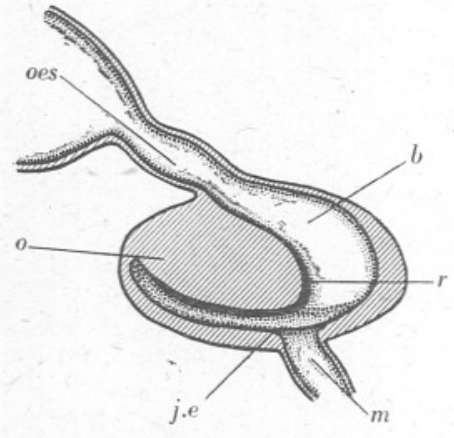

Fig. I. Calma. Diagrammatic view of the left half of the buccal mass. $b$, buccal cavity; $j . e$, biting edge of jaw; $m$, mouth; $o$, odontophore; oes, oesophagus; $r$, radula. caused by the transverse muscles which stretch between the two sides of the odontophore (Fig. $2 \mathrm{~A}, t .0$ ). Thus the three sharp biting edges come together and nip the egg membranes between them. Pressure is exerted by the oral hood (Evans, I922) and the contents of the egg are sucked through the slits into the capacious stomach or 'gastric sac' from which diverticula run into the cerata. Here storage, digestion and absorption take place. There is no anus. Little indigestible waste material is present, the amount depending on the stage of development of the embryos eaten, and the residue from the meals accumulates slowly, the 'fine brown granules' being compressed into a mass which does not mix with the next meal. Apparently this accumulation is never sufficient to inconvenience the animal.

\section{NEBALIOPSIS TYPICA}

As already mentioned the feeding of Nebaliopsis cannot be observed, and thus its mechanism must necessarily be hypothetical. The accumulated evidence suggests that the process is as follows.

An egg is gripped by means of the long recurved setae on the mandibular palps (Cannon, I93I) and held close to the mouth. The dilator muscles on the walls of the oesophagus and the dorsal side of the foregut provide the suction necessary to draw part of the egg through the mouth. The action of these muscles also draws apart the heavily chitinized biting surfaces of the 
'lateral thickenings' and the 'dorsal ridge'. The mandibles are soft and useless for biting, thus the egg is not punctured outside the mouth. The 'plated' walls of the oesophagus are then brought together by the circular muscles of this region and grip the slippery surface of the egg, while the
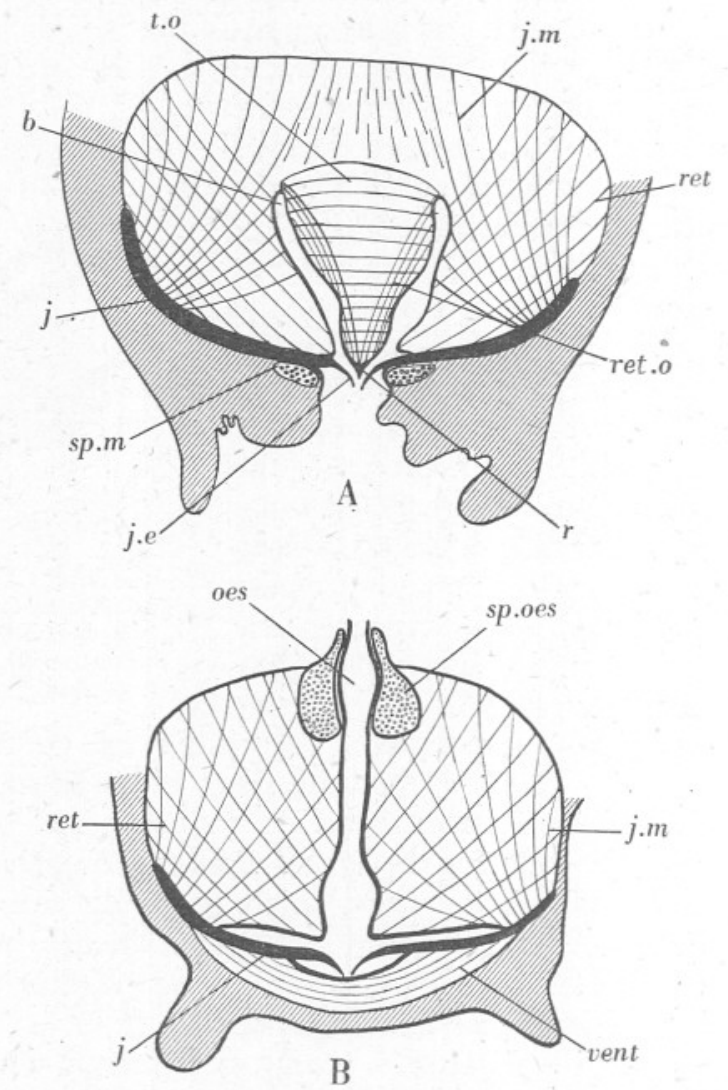

Fig. 2. Calma. A, semi-diagrammatic section of the buccal mass passing through the mouth and odontophore. B, semi-diagrammatic section of the buccal mass anterior to section A and passing through the oesophagus. $b$, buccal cavity; $j$, jaw; j.e, biting edge of jaw; $j . m$, muscles attached to the angle of the jaw; oes, oesophagus; $r$, radula; ret, retractor muscles of the buccal mass; ret.o, retractor muscles of the odontophore; sp.m, sphincter muscles of the mouth; sp.oes, sphincter muscles of the oesophagus; $t .0$, transverse muscles of the odontophore; vent, ventral transverse muscles.

circular muscles of the cardiac and pyloric regions of the foregut cause a biting action of the dorsal ridge against the lateral thickenings. Thus two slits are cut in the egg membranes through which the contents of the egg can be sucked. This then passes into the large digestive sac for storage, digestion and absorption. Digestive glands open into the anterior end of this sac by a number of small apertures. The intestine has an exceedingly narrow lumen 
and leads from the anterior end of the digestive sac to the anus. It is very improbable that much waste matter will pass through it as, in addition to the narrowness mentioned, there are no suitable muscles to force evacuation of the blind digestive sac.

\section{COMPARISON AND DISCUSSION \\ Method of attacking eggs}

Fish eggs are very slippery objects and must be firmly gripped. Calma applies its 'face' tightly to the egg while Nebaliopsis probably uses its mandibular palps.
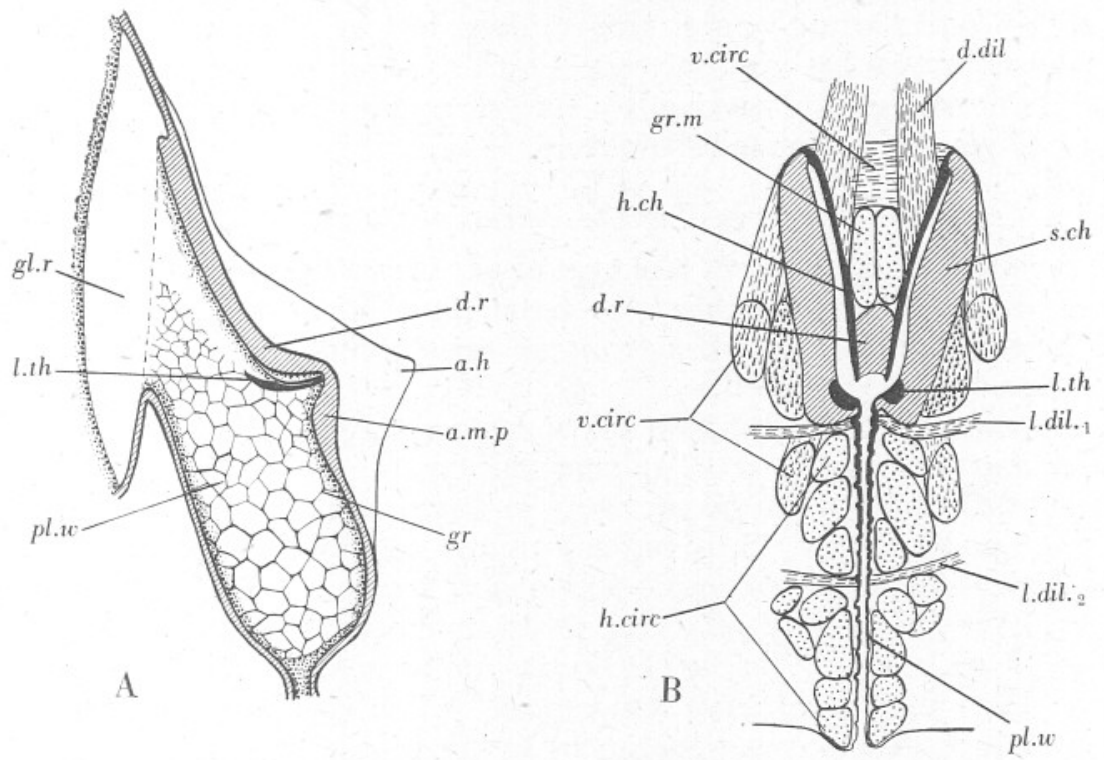

Fig. 3. Nebaliopsis. A, diagrammatic view of the left half of the fore-gut. B, semi-diagrammatic section of the oesophagus and foregut. a.h, anterior horn; a.m.p, anterior median projection; d.dil, dorsal dilator muscles; d.r, dorsal ridge; gl.r, glandular region; $g r$, grooves in chitin; gr.m, groove muscles; $h . c h$, hard chitin of dorsal ridge; $h . c i r c$, horizontal circular muscles; l.dil..$_{1}$, l.dil.2, lateral dilator muscles of the oesophagus; l.th, lateral thickenings; pl.w, plated walls of the oesophagus; s.ch, soft chitin of lateral walls; v.circ, vertical circular muscles.

\section{Piercing of the egg membranes}

The egg once 'caught' is too large and slippery a sphere to be swallowed whole by either of the animals concerned, but it cannot be chewed in the usual way lest the liquid or semi-liquid contents be washed away and wasted. The membranes must therefore be pierced in such a position that there is little chance of loosing the contents of the egg. The structures concerned are extraordinarily similar in design and homologous in function.

In each case a part of the membranes is sucked in through the mouth and gripped by corrugated surfaces. In Calma these are formed by folds of the skin around the mouth and in Nebaliopsis by the curious 'crazy-paving' of 
the chitin of the oesophagus which forms plates over each cell and grooves over each intercellular junction (Fig. $3 \mathrm{~A}, \mathrm{gr}$ ).

The similarity of outline of the cavities into which the membranes are drawn is obvious from a comparison of Figs. 2 A and 3 B. Both Calma and Nebaliopsis have one median and two lateral hard biting processes with deep channels between them in which folds of the egg membranes can lie. In Calma each of these processes has a sharp edge so that when the jaws come together and the radula meets them three slit-like incisions will probably be made. (Note. The sharp edges of the jaws appear to be equivalent to the 'strongly cuticularized ventral groove' described by Evans.) In Nebaliopsis the dorsal process has two sharp edges each of which bites against one of the lateral thickenings thus making two slits, or perhaps four if the double folds of membrane are perforated completely.

\section{Removal of the contents of the eggs}

Calma has been shown to take eggs at all stages of development. Without more material it is impossible to be certain on this point in Nebaliopsis, but the homogeneity of the food mass in the two specimens examined indicates that yolk only is swallowed.

The yolk or young embryos can be sucked through the slits in the egg membranes and passed to the storage chamber by the action of the musculature of the walls of the buccal cavity in Calma and of the oesophagus and stomach in Nebaliopsis. These muscles are the same as those used to produce the original suction on the membranes and to bring the biting parts together. In addition Calma has a sphincter muscle round the oesophagus which will prevent back suction from the gastric sac. In Nebaliopsis the circular muscles probably contract in turn, not simultaneously, thus causing a wave of motion such as is visible in living specimens of Nebalia bipes. This action would direct the flow of fluid in the correct direction.

\section{Storagé}

Suitable eggs are only found at certain seasons of the year and therefore both Calma and Nebaliopsis must be prepared to take in large quantities of food while it is available and store it while it is slowly digested and utilized as required.

In Calma a definite cycle of feeding and reproduction has been demonstrated. The large gastric sac provides the storage chamber. This becomes enormously distended during the feeding period till it almost completely fills the body, while diverticula from it stretch into the cerata which also become swollen.

Nebaliopsis similarly has an enormous storage sac which like that of Calma almost completely fills the body. Owing to the chitinized body wall there is a limit to the distension possible and the size of the sac is probably maintained even when empty. 


\section{Digestion and absorption}

In Calma salivary glands are present with ducts opening into the buccal cavity at the posterior ventral edges of the lateral pads, and the epithelium lining the cerata is also glandular. Secretion from these glands passes on to the food and causes partial digestion in the gastric sac. The thick fluid which results from this process passes into the cerata for final digestion and absorption.

In Nebaliopsis glands open into the anterior end of the digestive sac. Thus their secretion can be added to the food as it enters the sac and be mixed with it before storage so that continuous slow digestion of the whole food mass is possible. Absorption appears to take place over the entire surface of the sac.

\section{Evacuation of waste}

In correlation with the fact that fish eggs contain very little that is indigestible Calma has no anus and allows the small amount of residual matter to accumulate, while Nebaliopsis has such an extremely narrow intestinal lumen that it is doubtful whether waste matter could pass along it. In addition the attachment of the intestine to the anterior end of the large blind diverticulum and the absence of suitable musculature for the evacuation of the diverticulum make the use of the intestine most improbable.

\section{CoNCLUSION}

The typical Eolid feeds by scratching particles of food from a solid surface by means of the radula. The conversion of this scratching action into a piercing one involves little structural change. The typical Nebaliacean, however, is a filter feeder and considerable adaptation is necessary to produce the piercing action. In both groups the typical forms feed almost continuously and prolonged storage of food is unnecessary, while in the specialized egg feeders storage is essential and cavities are provided for this purpose.

To the continuous feeders, whose food contains much indigestible material, evacuation of waste is essential, while in the egg feeders this is not so.

Thus the characters in which Calma glaucoides and Nebaliopsis typica differ from typical members of their groups may be associated with their specialized diet. Functional necessity has made the arrangements extraordinarily similar in plan in spite of the immense general difference between a mollusc and a crustacean.

\section{REFERENCES}

Cannon, H. G., I93I. Nebaliacea. Discovery Reports, Vol. III, pp. I99-222.

Evans, T. J., I922. Calma glaucoides-a study in adaptation. Quart. Fourn. Micr. Sci., Vol. LXVI, pp. 439-55.

RowetT, H. G. Q., I943. The gut of the Nebaliacea. Discovery Reports, Vol. xxIII, pp. I-I8. 